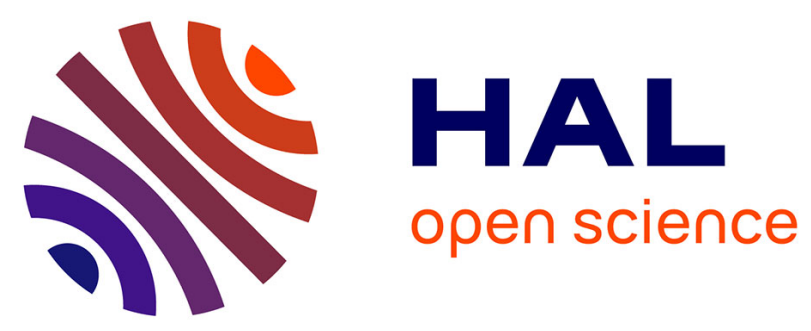

\title{
Interference-aware Frame Optimization for the Return Link of a Multi-Beam Satellite
}

\author{
Yoann Couble, Emmanuel Chaput, Thibault Deleu, Cédric Baudoin, \\ Jean-Baptiste Dupé, Caroline Bés, André-Luc Beylot
}

\section{To cite this version:}

Yoann Couble, Emmanuel Chaput, Thibault Deleu, Cédric Baudoin, Jean-Baptiste Dupé, et al.. Interference-aware Frame Optimization for the Return Link of a Multi-Beam Satellite. IEEE International Conference on Communications (ICC 2017), May 2017, Paris, France. 10.1109/ICC.2017.7996645 . hal-03109259

\section{HAL Id: hal-03109259 \\ https://hal.science/hal-03109259}

Submitted on 19 Jan 2021

HAL is a multi-disciplinary open access archive for the deposit and dissemination of scientific research documents, whether they are published or not. The documents may come from teaching and research institutions in France or abroad, or from public or private research centers.
L'archive ouverte pluridisciplinaire HAL, est destinée au dépôt et à la diffusion de documents scientifiques de niveau recherche, publiés ou non, émanant des établissements d'enseignement et de recherche français ou étrangers, des laboratoires publics ou privés. 


\title{
Interference-aware Frame Optimization for the Return Link of a Multi-Beam Satellite
}

\author{
Yoann Couble ${ }^{1}$, Emmanuel Chaput ${ }^{2}$, Thibault Deleu ${ }^{3}$, Cédric Baudoin $^{3}$, Jean-Baptiste Dupé ${ }^{4}$ \\ Caroline Bès ${ }^{4}$ and André-Luc Beylot ${ }^{2}$ \\ ${ }^{1}$ yoann.couble@enseeiht.fr, Université de Toulouse; INP; TéSA; F-31017 Toulouse, France \\ 2 chaput,beylot\}@enseeiht.fr, Université de Toulouse; INP; IRIT; F-31017 Toulouse, France \\ 3 thibault.deleu,cedric.baudoin\}@ thalesaleniaspace.com, Thales Alenia Space, France \\ ${ }^{4}\{$ jean-baptiste.dupe,caroline.bes $\} @$ cnes.fr, CNES, France
}

\begin{abstract}
High throughput satellites have proven to be an excellent solution to provide Internet services to white spots or to complement other existing infrastructures. With the use of multi-beam antennas, the same frequency may be reused dozens of times across a single satellite coverage area, increasing the system capacity and profitability.

Legacy frequency reuse patterns such as uncoordinated 4-color scheme ensure interference isolation at the expense of important capacity reduction, using only one fourth of the operator's share in the scarce Ka band. Thus, to increase the per-beam available bandwidth, it is necessary to look for more aggressive and efficient Frequency Reuse schemes, generating higher CoChannel Interference which has to be analyzed and handled. In this paper, we focus on the DVB-RCS2 return link of a multi-beam satellite and investigate the possibility of reaching the upper bound of a coordinated 2-color system capacity through the use of Interference-aware User Scheduling techniques. We first formalize the problem as an Integer Linear Program and study the impact of greedy simplifications on the optimality and processing times of our optimization models.
\end{abstract}

\section{INTRODUCTION}

With the development of High Throughput Satellite and Multiple Beam Antennas, the potential amount of clients accessing simultaneously the satellite has increased drastically. However, there are many challenges to overpass before reaching this new potential, especially at the MAC layer to avoid or to deal with new risks of collisions. In ETSI's DVB-RCS2 [1], Multiple Frequency Time Division Multiple Access (MFTDMA) combined with Demand Assigned Multiple Access (DAMA) is used as the access scheme for reliable data transmissions. Using more numerous narrow beams means more aggregated bandwidth for the same coverage, but also a more dense usage of each uplink frequency. Reusing a subcarrier in two beams close to each other generates higher Co-Channel Interference (CCI) which is not entirely mitigated by the antenna directivity.

The first and straightforward solution, used for dimensioning current systems, is to use static frequency reuse patterns, dedicating a fraction of the available bandwidth to a subset of the beams, in a geometrically regular way. Frequency Reuse $\mathbf{X}(\mathrm{FRX})$ schemes (reusing a frequency band in one out of $\mathbf{X}$ beams, with commonly $\mathbf{X} \in 3,4,7$ ) have proven to be easy to deploy but at the cost of a low per-beam bandwidth, since only $1 / \mathbf{X}$-th of the bandwidth is available in each beam. Then, Full or Fractional frequency reuse schemes define a central area where sub-carriers are reused in every beam, i.e. FR1, plus an FRX scheme for the edge areas. These schemes have been extensively studied in the terrestrial networks, thoroughly presented in [2], and sometimes adapted to satellite systems.

Yet, static frequency reuse schemes are based on the estimation of the worst-case scenario, which limits their flexibility in several ways:

- Between colors, static bandwidth allocation means less flexibility in case of demand fluctuation.

- Inside a color, the worst case interference estimation will cause a terminal to use a less efficient Modulation and Coding scheme (ModCod) when in reality, it would have been possible to use a higher order ModCod.

In other words, there are plenty of situations where resources could be used more greedily. However, introducing flexibility through the use of Dynamic Inter Beam Interference Coordination (D-IBIC) requires a high degree of coordination between users and gateways, as well as solving the User Scheduling Problem which is well known to be NP-hard.

This paper intends to model and analyze the achievable improvements possible thanks to D-IBICs on the Return Link (RL). In section II, we will briefly review and comment existing coordinated interference management schemes. Then in section III we model and formulate the problem of globally allocating MF-TDMA bursts to users. The problem complexity and scalability are also discussed in section IV. In section V we present our numerical results and analyze the impact of several chosen approximations on the performance.

\section{DYNAMIC INTERFERENCE COORDINATION}

Using D-IBIC in the Forward Link (FL) (resp. RL) requires a Channel State Information (CSI) exchange mechanism to inform the gateway of the reception (resp. transmission) condition and interference levels experienced by users. Obtaining CSI is then at the heart of D-IBIC in the FL (resp. RL), requiring thorough studies to find the best tradeoff between CSI consistency and generated signaling traffic. Then, the next issue is to use this CSI to perform an optimal scheduling of the S2/S2X BBFRAMES (resp. MF-TDMA Frame). In the FL, 

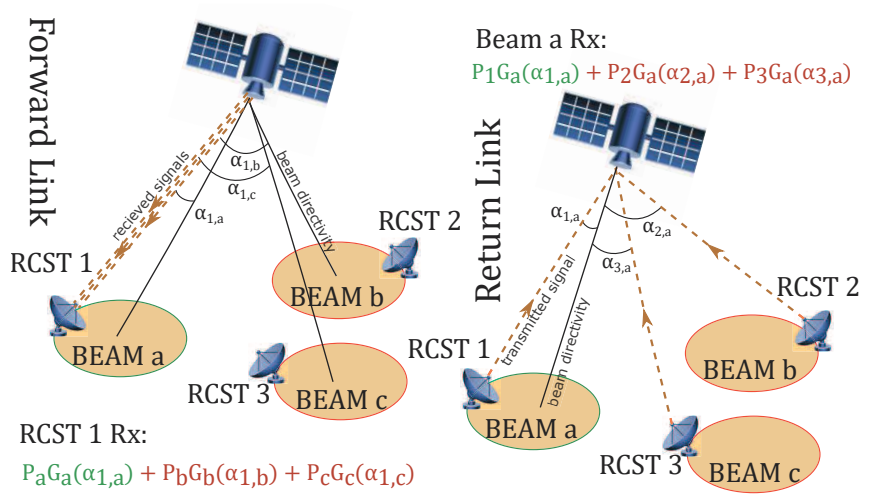

Fig. 1. Interference in the Forward Link and Return Link

[3], [4] and [5] among others tackle this issue with different approaches, the former by successively allocating sub-carriers and then optimizing the power for each while the latter uses a heuristic benefiting from the beam-hopping flexibility.

What differs between the FL and RL in solving this problem is the nature of interference. Indeed, as shown in Fig 1, interference power in the FL only depends on the user's position in each beam antenna pattern. Whereas in the RL, the interference experienced by a user is given by the interfering users position in the interfered user beam antenna pattern. In other words, the interference generated in the FL doesn't rely on which user is scheduled, when in the RL it is at the heart of the problem. This schedule/interference enslavement brings a great diversity which may be used to improve further the system throughput, along with a huge combinatorial complexity.

There is a lot of literature around this kind of issues in terrestrial networks [6], whereas there are only a few studies on the matter for the RL of a DVB-S2/RCS2 system. First, RCS/RCS2 frame optimization has been studied in, among others, [7] and [8], but without considering interference management.

The authors in [2] propose a simple scheduling heuristic aiming to improve the minimum Carrier power to Interference power ratio $(C / I)$. The authors focus on frame reorganization and assume that users have already been assigned a number of Bandwidth Time Unit (BTU) and that all the Super Frame BTUs will be allocated. This approach has numerous practical advantages such as being able to implement it without modifying the user-BTU allocation algorithm. On the other hand, they don't take ModCod into account, nor do they deal with the user-BTU allocation. Additionally, in many cases, it may be more interesting to leave a few BTU unused (especially when FR1 or FR2 schemes are used) so that other users experience a better $C / I$ and may use more efficient ModCods. In [9] the Multi-user MIMO (Mu-MIMO) paradigm is used to tackle the uplink user scheduling problem. Beams are clustered by groups of 7, and Successive Interference Cancellation (SIC) is used to combine the seven simultaneous user signals. User scheduling is then performed through the use of multi-

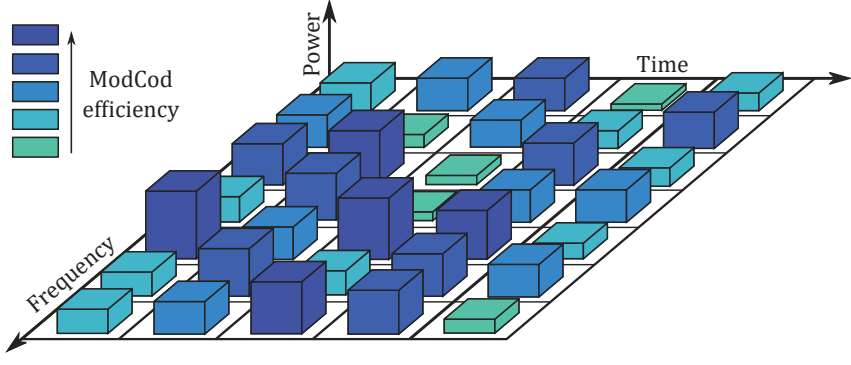

Fig. 2. DVB-RCS2 MF-TDMA Structure, with individual ModCod setting depending on the reception power

partite graph matching. [4] pushes further these principles and puts forward multiples algorithms to perform interference aware user scheduling based on an FR1 system. While these algorithms offer large gains (from $+50 \%$ without SIC to $+99 \%$ with SIC throughput vs. FR4-random scheduling), their bandwidth efficiency is not so high since they need $+300 \%$ bandwidth for that. Moreover, [9] and [4] do not allow more than one empty slot which, as we will show in this paper, may not benefit the system.

\section{System Model AND Formalization}

\section{A. System Model}

We consider a system composed of a Gateway, a transparent multi-beam satellite and RCS Terminals (RCST). The satellite has $N_{\text {beams }}$ directive antennas, defining $N_{\text {beams }}$ beams. The beams are organized in a hexagonal grid and split over the two polarizations.

To simplify the notations and modelisation, we will limit our study to one of the polarization. The models and optimization that we propound thereafter can be applied to either polarization in the same way.

Each beam $k$ has a set of RCST $\left\{1, \ldots, i_{k}, \ldots, N_{\text {users }}(k)\right\}$, able to transmit using a set of ModCods defined in Table II. We consider a given DVB-RCS2 MF-TDMA frame, composed of $N_{t t i}$ timeslots and $N_{\text {carriers }}$ equal bandwidth subcarriers. We call BTU (timeslots, sub-carrier) couples. In this paper we concentrate on CCI, and will treat adjacent channel interference (ACI) and cross-polarization interference (CPI) as constant noises. As shown in Fig. 2, DVB-RCS2 allows the use of different ModCod independently on every BTU, which is an important degree of freedom to cope with different transmission conditions.

\section{B. Problem Formulation}

First we introduce the binary variables $x_{i_{k}, m}^{t, c}$ to indicate whether RCST $i_{k}$ is transmitting on BTU $(t, c)$ with ModCod $m$ or not.

1) User Link Budget: For each RCST $i_{k}$, transmitting with ModCod $m$ on a BTU $(t, c)$, we use the following Signal to Noise plus Interference Ratio (SNIR) expression:

$$
\operatorname{SNIR}\left(i_{k}, m\right)=\frac{P_{i_{k}} G_{k}\left(i_{k}\right)}{N+\sum_{\substack{k^{\prime} \neq k \\ j_{k^{\prime}}, m^{\prime}}} x_{j_{k^{\prime}}, m^{\prime}}^{t, c} P_{j_{k^{\prime}}} G_{k}\left(j_{k^{\prime}}\right)}
$$


TABLE I

NotATIONS

\begin{tabular}{|l|l|}
\hline Notation & Description \\
\hline $1 \leq k \leq N_{\text {beams }}$ & Beam identifier \\
\hline $1 \leq m \leq N_{\text {modcods }}$ & ModCod identifier \\
\hline $1 \leq t \leq N_{t t i}$ & Time transmit interval (TTI) \\
\hline $1 \leq c \leq N_{\text {carriers }}$ & Sub-carrier identifier \\
\hline $\mathcal{I}(k)$ & Set of users in beam $k$ \\
\hline$i_{k} \in \mathcal{I}(k)$ & User identifier in beam $k$ \\
\hline$x_{i_{k}, c}^{t, c}$ or $x_{i_{k}, m}^{c}$ & Binary variable \\
\hline$P_{i_{k}}$ & Transmit power for user $i_{k}$ \\
\hline$G_{k^{\prime}}\left(i_{k}\right)$ & Reception gain of beam $k^{\prime}$ for user $i_{k}$ \\
\hline$N$ & Sub-carrier noise power (incl. ACI/CPI) \\
\hline$\Gamma_{m}^{t h r e s h}$ & Threshold SNIR for modcod $m$ \\
\hline$r_{m}$ & Instant throughput for modcod $m$ \\
\hline
\end{tabular}

where $P_{i_{k}}$ is the transmission power for user $i_{k}$ of beam $k, G_{k^{\prime}}\left(i_{k}\right)$ is the reception gain of beam $k^{\prime}$ for user $i_{k}$. $G_{k^{\prime}}\left(i_{k}\right)$ takes into account the antenna gain, its directivity but also every other signal attenuation phenomenon like antenna pointing error, atmospheric losses, path loss, etc. Here $N$ incorporates the thermal noise, and the ACI and CPI noises.

A transmission is only useful if it achieves the SNIR threshold $\left(\Gamma_{m}^{\text {thresh }}\right.$, see Table II) of the ModCod $m$ it is transmitting at, i.e. if $\operatorname{SNIR}\left(i_{k}, m\right) \geq \Gamma_{m}^{\text {thresh }}$. Any additional SNIR margin will not increase the throughput, except if it overpasses the next ModCod threshold.

TABLE II

Modulation And Coding Schemes

\begin{tabular}{|c|c|c|}
\hline ModCod & SNIR threshold & Throughput \\
\hline QPSK-1/3 & $-0.5 \mathrm{~dB}$ & $0.984 \mathrm{Mbps}$ \\
\hline QPSK-5/6 & $5.9 \mathrm{~dB}$ & $2.664 \mathrm{Mbps}$ \\
\hline 8PSK-3/4 & $8.8 \mathrm{~dB}$ & $3.200 \mathrm{Mbps}$ \\
\hline 8PSK-5/6 & $10.2 \mathrm{~dB}$ & $3.552 \mathrm{Mbps}$ \\
\hline 16QAM-5/6 & $12 \mathrm{~dB}$ & $4.792 \mathrm{Mbps}$ \\
\hline
\end{tabular}

In the coming sections, the link budget expression is reworked in order to formulate an Integer Linear Program, taking advantage of these SNIR margins to maximize the resource utilization and ultimately the system throughput.

2) SNIR constraint formulation: As used in [10], we rearrange the terms in equation (1) combined with the SNIR threshold inequality to obtain the following condition to the successful transmission for any user $i_{k}$ using $\operatorname{ModCod} m$ :

$$
\frac{P_{i_{k} G} G_{k}\left(i_{k}\right)}{\Gamma_{m}^{\text {thresh }}} \geq N+\sum_{\substack{k^{\prime} \neq k \\ j_{k^{\prime}}, m^{\prime}}} x_{j_{k^{\prime}}, m^{\prime}}^{t, c} P_{j_{k^{\prime}}} G_{k}\left(j_{k^{\prime}}\right)
$$

This constraint must be verified when BTU $(t, c)$ of beam $k$ is assigned to RCST $i_{k}$ for a transmission with ModCod $m$. However, when this BTU is not assigned to user $i_{k}$, the inequality constraint (2) has no meaning whatsoever and must be ignored. Hence, we introduce a sufficiently large number B to ensure that the inequality constraint (2) is always true when $x_{i_{k}, m}^{t, c}=0$. So, $\forall k, i_{k}, t, c, m$ :

$$
\begin{aligned}
x_{i_{k}, m}^{t, c} \cdot \frac{P_{i_{k}} G_{k}\left(i_{k}\right)}{\Gamma_{m}^{t h r e s h}} \geq & \left(x_{i_{k}, m}^{t, c}-1\right) \cdot \mathbf{B}+N \\
& +\sum_{\substack{k^{\prime} \neq k \\
j_{k^{\prime}}, m^{\prime}}} x_{j_{k^{\prime}}, m^{\prime}}^{t, c} P_{j_{k^{\prime}}} G_{k}\left(j_{k^{\prime}}\right)
\end{aligned}
$$

When $x_{i_{k}, m}^{t, c}=1$, the constraint is equivalent to (2). And when $x_{i_{k}, m}^{t, c}=0$, the $\left(x_{i_{k}, m}^{t, c}-1\right) \cdot \mathbf{B}$ term guarantees a negative right term to satisfy the constraint.

3) Global Frame Optimization Problem: From constraint (3d) and the system instant throughput maximization objective, we formulate the following Integer Program:

$$
\begin{aligned}
& \text { Problem GFO: } \\
& \underset{\mathbf{X}}{\operatorname{Maximize}} F(\mathbf{X}) \\
& \forall k, t, c \quad \sum_{i_{k}, m} x_{i_{k}, m}^{t, c} \leq 1 \\
& \forall k, i_{k}, t \quad \sum_{c, m} x_{i_{k}, m}^{t, c} \leq 1 \\
& \forall k, i_{k}, \quad x_{i_{k}, m}^{t, c} \cdot \frac{P_{i_{k}} G_{k}\left(i_{k}\right)}{\Gamma_{m}^{t h r e s h}} \geq\left(x_{i_{k}, m}^{t, c}-1\right) \cdot \mathbf{B}+N \\
& m, t, c \quad+\sum_{\substack{k^{\prime} \neq k \\
j_{k^{\prime}}, m^{\prime}}} x_{j_{k^{\prime}}, m^{\prime}}^{t, c} P_{j_{k^{\prime}}} G_{k}\left(j_{k^{\prime}}\right) \\
& \begin{array}{l}
\forall k, i_{k}, \\
m, t, c
\end{array} \quad x_{i_{k}, m}^{t, c} \in\{0,1\}
\end{aligned}
$$

where $\mathbf{X}$ is the vector of all $x_{i_{k}, m}^{t, c}$ and $F(\mathbf{X})$ is an objective function to maximize the system throughput while guaranteeing fairness among users. The first constraint ( $3 b)$ comes from the MF-TDMA frame structure: there cannot be more than one RCST transmitting on a BTU, whichever the ModCod. We consider here relatively simple terminals, with only one transmission chain, as expressed in constraint (3c)

\section{DECOMPOSITION AND DERIVED MODELS}

\section{A. Time-wise decomposition}

The GFO problem may be non-linear, has a large amount of variables and its solution space has a lot of symmetries. In the following, we propose to decompose it in $N_{t t i}$ identical subproblems, to be treated on a timeslot to timeslot basis rather than at the frame level, with a causality relationship from one instance to the other.

While it may also remove a degree of freedom in the allocation, we expect that the loss of optimality is negligible and the gain of simplicity is important. Also, treating the whole frame at once would either produce $N_{t t i}$ times the same timeslotoptimized set of user or require a fairness constraint/utility function to limit the amount of timeslots received by a user. In the latter case, the fairness constraint/utility function would then interfere with a SuperFrame to SuperFrame long-term fairness mechanism implemented in the Gateway.

Moreover, the timeslot-local throughput maximization may not even be unfair as it tends to fulfill the demands more quickly and hence save resource to serve less "profitable" users. Anyhow, in this paper we focus on maximizing the 


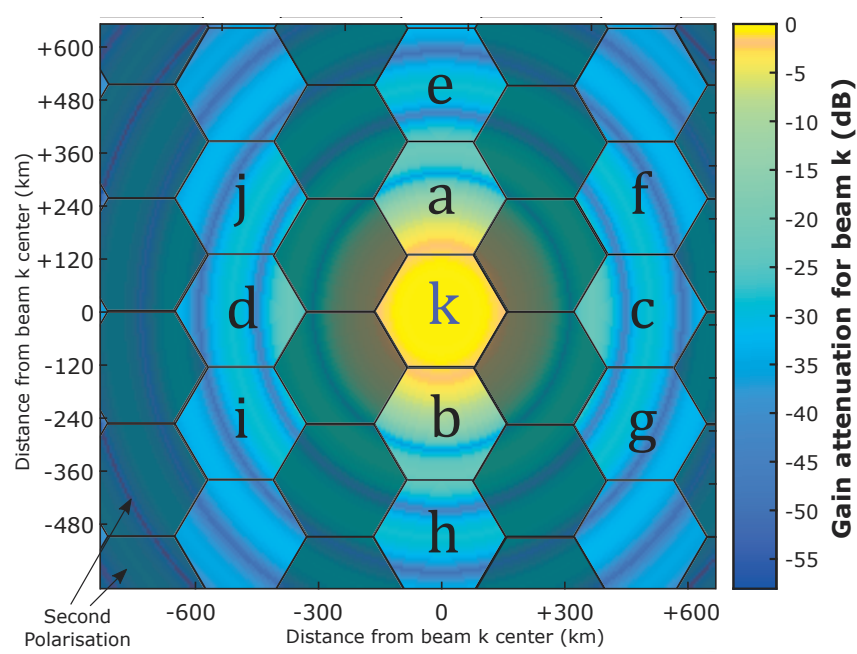

Fig. 3. Interference power generated by users located in beams of the same polarization as the reference beam $k$

system capacity by "filling" sub-carriers as much as possible, and to put the fairness aside for further works.

\section{B. Neighbor orders}

TABLE III

INTERFERING SET DEFINITION AND EMPIRICAL ESTIMATED INTERFERENCE FOR DIFFERENT SCHEMES.

\begin{tabular}{|l|c|r|}
\hline Restriction scheme $s$ & interfering beam set $\Omega_{s}(k)$ & $I_{\text {est }} / N$ \\
\hline order 1 & $\{a, b\}^{\dagger}$ & $-7.5 \mathrm{~dB}$ \\
\hline order $\leq 1.5$ & $\{a, b, c, d\}$ & $-12 \mathrm{~dB}$ \\
\hline order $\leq 2$ & $\{a, b, c, d, e, f, g, h, i, j\}$ & $-15 \mathrm{~dB}$ \\
\hline global & $\llbracket 1, N_{\text {beams }} \rrbracket \backslash\{k\}$ & - inf $\mathrm{dB}$ \\
\hline
\end{tabular}

${ }^{\dagger}$ Beam letters refer to Fig. 3

In Fig. 3 we can see that most of the interference experienced by a beam comes from RCST located in a very limited set of beams, and that after a certain distance, the interference generated by RCSTs can reasonably be neglected or at least estimated and considered as constant noise. Beams $a$ and $b$ are the most impacting beams and should thus always be taken into account in the link budget. Second order beams ( $c, d$ and $e, f, g, h, i, j)$ are less influential, but their cumulative interference power should not be neglected.

Let us call $\Omega_{s}(k) \subset \llbracket 1, N_{\text {beams }} \rrbracket \backslash\{k\}$ the restricted set of beams which will be accounted in further alternate versions of the problem, where $s$ is the restricted scheme of accounted neighbors. For these restricted models, we ignore users outside of the considered beams, meaning that errors in the allocation may happen. It is thus necessary to estimate the interference $I_{\text {est }}$ generated by the ignored beams, and to include it in the link budget constraint (3d). In this section, we will consider the interfering beam sets and corresponding (empirically determined) estimated interference defined in Table III.

As a consequence, we define a collection of TimeSlot Optimisation (TSO $(s)$ ) Integer Linear Programs, one for each restriction scheme $s$ that we will solve and compare in the next section.

$$
\begin{aligned}
& \text { Problem TSO }(s) \text { : } \\
& \underset{\mathbf{X}}{\operatorname{Maximize}} \sum_{k, i_{k}} \sum_{c, m} x_{i_{k}, m}^{c} r_{m} \\
& \text { s.t.: } \\
& \forall k, c \quad \sum_{i_{k}, m} x_{i_{k}, m}^{c} \leq 1 \\
& \forall k, i_{k} \quad \sum_{c, m} x_{i_{k}, m}^{c} \leq 1 \\
& \forall k, i_{k}, \quad x_{i_{k}, m}^{c} \frac{P_{i_{k}} G_{k}\left(i_{k}\right)}{\Gamma_{m}^{t h r e s h}} \geq\left(x_{i_{k}, m}^{c}-1\right) \cdot \mathbf{B}+N \\
& m, c \\
& +I_{e s t}+\sum_{\substack{k^{\prime} \in \Omega_{s}(k) \\
j_{k^{\prime}}, m^{\prime}}} x_{j_{k^{\prime}}, m^{\prime}}^{c} P_{j_{k^{\prime}}} G_{k}\left(j_{k^{\prime}}\right)
\end{aligned}
$$

$$
\begin{aligned}
& \forall k, i_{k}, \\
& m, c
\end{aligned} x_{i_{k}, m}^{c} \in\{0,1\}
$$

where $r_{m}$ is the instant throughput of MocCod $m$.

\section{Sub-carrier-wise decomposition}

Following the same approach as previously, we now consider the possibility of dividing the problem into $N_{\text {carriers }}$ sub-problems. Yet, contrarily to the time-wise decomposition, the $N_{\text {carriers }}$ sub-problems are not independent. To cope for the absence of constraint (4c), solving these sub-problems must be done sequentially, and users having received an allocation in the $c$-th resolution must be removed from the user pool that $(c+1)$-th instance of segC-TSO $(s)$ takes as input.

Hence, we define a second collection of sequential subCarrier, TimeSlot Optimization ( seqC-TSO $(s)$ ) Problems, defined by a sequence of Integer Linear sub-Programs, as shown in Eq. (5) and Algorithm 1:

Sub-problem sub-seqC-TSO $(s)$ :

$$
\begin{aligned}
\underset{\mathbf{X}}{\operatorname{Maximize}} & \sum_{k, i_{k}} \sum_{m} x_{i_{k}, m} r_{m} \\
\text { s.t.: } & \\
\forall k & \sum_{i_{k}, m} x_{i_{k}, m} \leq 1 \\
& x_{i_{k}, m} \frac{P_{i_{k}} G_{k}\left(i_{k}\right)}{\Gamma_{m}^{\text {thresh }} \geq} \geq\left(x_{i_{k}, m}-1\right) \cdot \mathbf{B}+N \\
\forall k, i_{k}, m & \quad+I_{\text {est }}+\sum_{\substack{k^{\prime} \in \Omega_{s}(k) \\
j_{k^{\prime}}, m^{\prime}}} x_{j_{k^{\prime}}, m^{\prime}} P_{j_{k^{\prime}}} G_{k}\left(j_{k^{\prime}}\right)
\end{aligned}
$$

$\forall k, i_{k}, m \quad x_{i_{k}, m} \in\{0,1\}$

\section{NumERICAL RESUltS}

A. Test case

We consider a hexagonal grid with $2 \times 15$ beams equally distributed between the two polarizations. In each beam, 


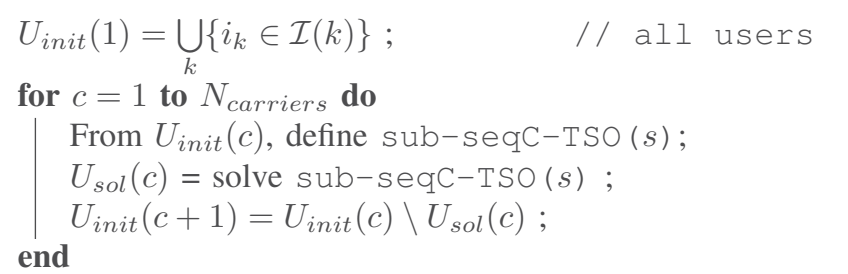

Algorithm 1: seqC-TSO $(s)$ definition

$N_{\text {users }}$ active users are placed uniformly across the beam coverage. Each active user is assumed to transmit with the same transmission power $P_{T x}$. Users are able to use any ModCod in Table II on any of the $N_{\text {carriers }}$ sub-carriers available for each beam in its beam polarization.

\section{B. Test methodology}

For a given scenario, TSO $(s)$ and seqC-TSO $(s)$ problems formulation are performed and numerically solved using Gurobi ILP optimizer [11] Gurobi ILP optimizer is configured to use a branch and bound algorithm. As a comparison reference, we also performed a 4 color scheme optimization with the same objective, where the interference is estimated in the worst case scenario. This reference is what is classically done in current systems. In each test, the same process is repeated 50 to 200 times (depending on the test case) with reinitialized users location to narrow the $95 \%$ confidence intervals. Tests data is described in Table IV.

TABLE IV

TEST DATA

\begin{tabular}{|l|l|l|l|}
\hline Parameter & Value & Parameter & Value \\
\hline \hline Beam aperture $\theta_{3 d B}$ & $0.27 \mathrm{deg}$ & Pointing error & $0.035 \mathrm{deg}$ \\
\hline RCST max gain & $50.5 \mathrm{dBi}$ & RCST power & $1 \mathrm{~W}$ \\
\hline Satellite max gain & $20.9 \mathrm{dBi}$ & Frequency band & $\mathrm{Ka}(29.5 \mathrm{GHz})$ \\
\hline Sub-carrier Bw. & $21 \mathrm{MHz}$ & BTU size & $1616 \mathrm{symbols}$ \\
\hline$N_{\text {beams }}$ & 15 for each polarization \\
\hline \multicolumn{4}{|c|}{ Optimization parameters } \\
\hline Large number B & $10^{5}$ & Gurobi Tolerance & 0.05 \\
\hline Hardware & Intel Core i7-4790 $(3.60 \mathrm{GHz}), 16 \mathrm{~GB}$ RAM \\
\hline
\end{tabular}

\section{Test description and comments}

1) Test 1 - Performance gains: First, we compared the performance of the different model families in three situations $\left(\rho=N_{\text {users }} / N_{\text {carriers }}\right)$ :

- $\rho=0.5$ Cases where there are only a few active users compared to the amount of available sub-carriers. This may correspond to a system with few professional users with high bandwidth demand or to a low charge period.

- $\rho=1$ Cases where there are as many active user as subcarriers.

- $\rho=2$ Cases where there are a lot of active users compared to the available sub-carriers, which could correspond to a system with a huge number of users, or a high charge period.

The results of this test case are shown in Fig. 4 and Fig. 5. Performance is measured in terms of total system throughput

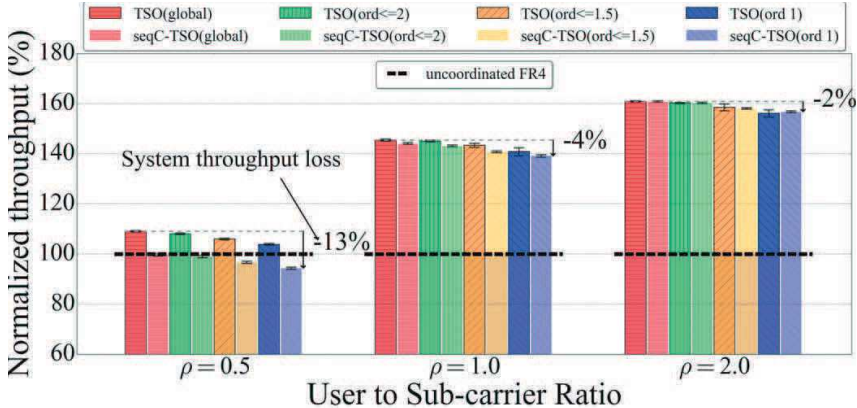

Fig. 4. Performance of the different models solution according to the user to sub-carrier ratio. Sub-carrier count is kept constant $\left(N_{\text {carriers }}=8\right)$ so that physical capacity is kept constant, while the user diversity varies with user count $\left(N_{\text {users }} \in\{4,8,16\}\right)$. ( $\left.N_{\text {tests }}=200\right)$

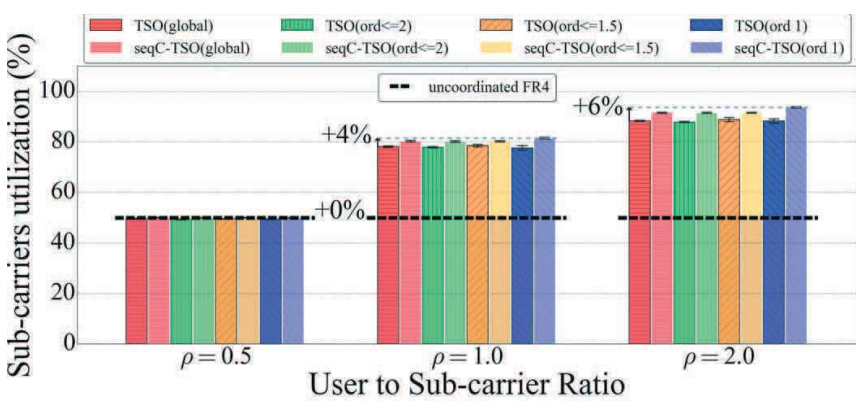

Fig. 5. Sub-carriers utilization according to the user to sub-carrier ratio.

relatively to the FR4 scheme with interference estimation. The first figure points out that neighbor restricted schemes (green, yellow and blue) perform fairly well compared to the global scheme (red), for both TSO (plain) and segC-TSO models (hatched), with only a $2 \%$ system throughput difference between TSO (global) and seqC-TSO (ord 1) at $\rho=2$.

A second observation is that, as could be expected, the segC-TSO problem family performs poorly for low values of $\rho$. Indeed when there are less active RCSTs than available subcarriers, seqC-TSO will not be able to spread RCST across the sub-carriers. Yet, as soon as there are more active RCST, the difference between TSO and seqC-TSO is negligible.

Fig. 4 also points out that the more active users, the higher the system throughput: spatial diversity increases with the amount of active users and more interesting RCST sets can be found. This also means that on average, we can expect that users will be able to use more efficient ModCods on their BTU and in the long term, this will increase the mean throughput per user. On Fig. 5 on the other hand, we represented the sub-carriers utilization. The FR4 reference scheme (black dashed line) is obviously limited to $50 \%$ of the polarization sub-carriers, while other schemes manage to use more. This confirms that in an FR4 scheme resources are under-utilized and that it is possible to use them more greedily. Also note that sub-carriers utilization is higher for seqC-TSO schemes, meaning that more users are served simultaneously, but with lower order ModCods. 


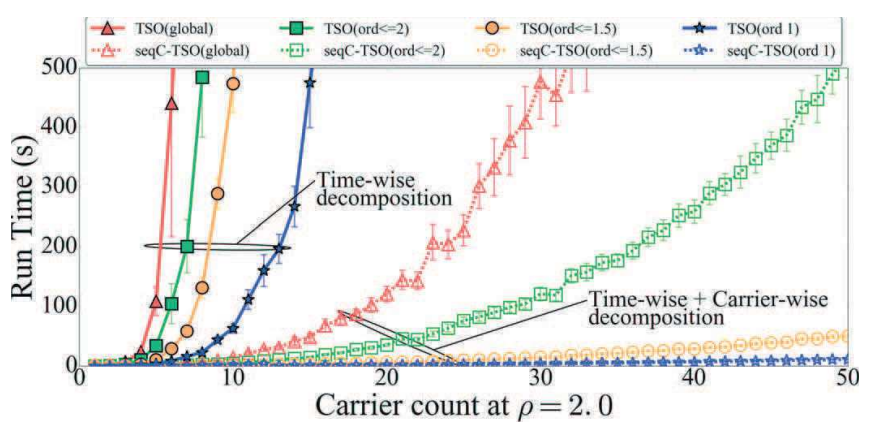

Fig. 6. Models computational complexity related to user and sub-carrier count. In this figure the user to sub-carrier ratio is kept constant to avoid effects highlited in Fig. 4. $\left(N_{\text {tests }}=50\right)$

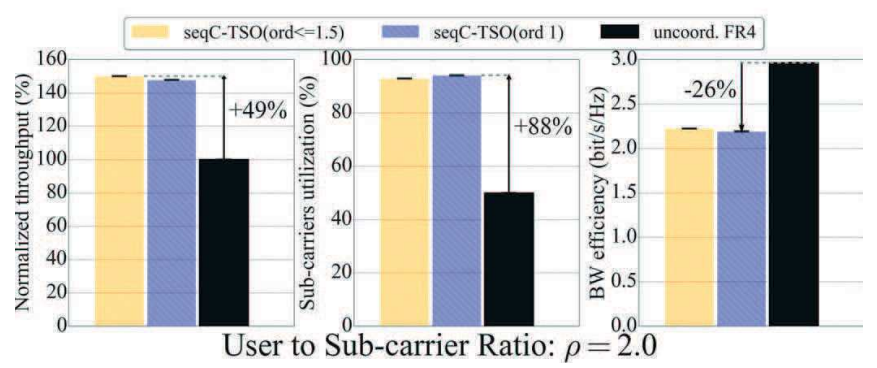

Fig. 7. Results for a larger scenario ( 90 beams, 30 carriers, 60 users per beam), comparing seqC-TSO (ord 1$)$, seqC-TSO (ord $\leq 1.5)$ and the reference FR4 scheme. The Bandwidth efficiency is also considered here. $\left(N_{\text {tests }}=\right.$ 100)

2) Test 2 - Scalability: Secondly, to emphasize the scalability of each formulation, we increased the problem size (i.e. sub-carrier count) progressively while maintaining the user to sub-carrier ratio $(\rho=2)$. Fig. 6 shows that for $\operatorname{TSO}(s)$ models it is quickly ( $N_{\text {carriers }} \approx 10$ ) impossible to even evaluate the upper bound of the system throughput, while for seqC-TSO $(s)$ models it is possible to reach much higher sizes of scenarios. In particular, seqC-TSO (ord $\leq 1.5)$ and seqC-TSO (ord 1) show promising resolution times, even though there is still a 1000-fold gap before being ready to use in practice.

3) Test 3 - Larger scenario: Additional simulations have been made for larger systems and solved for segC-TSO (ord 1), which offers a $+47 \%$ throughput increase compared to the FR4 scheme on systems with 90 beams, 30 active users per beam and 25 sub-carriers with a mean resolution time of 40s. Results are given in Fig. 7, showing consistent results, but with a slight loss of throughput partly due to the lack of precision in the interference estimation (especially for center beams). Last but not least, it is interesting to note that overall, using an FR2 scheme, even coordinated generates higher interference levels, meaning that the bandwidth efficiency is reduced: additional throughput comes at the expense of even larger bandwidth to carry on the feeder link.

\section{CONCLUSION AND FUTURE WORKS}

In this paper, we modeled the user scheduling problem through the use of an integer program, with a ModCodaware interpretation of the SNIR constraint. After discussing the relevance of decomposing the problem time-wise and carrier-wise, we studied the importance and contribution of different beam neighbors and defined several integer linear programs. Finally, we presented our numerical results, showing promising performances regarding system throughput and sub-carriers utilization.

The proposed simplifications presented very consistent results, with a low loss of optimality compared to the computational gains. Moreover, combined with a time and carrier-wise decomposition, the problem becomes solvable for real-scale systems.

Even though we managed to reduce drastically the computational complexity for solving the user scheduling problem, The complexity of the proposed methods should still be reduced before considering a real-time (slot-by-slot) implementation. However, our observations and conclusions remain valuable to formulate new heuristics and scheduling algorithms, as well as to combine our methods with advanced signal processing techniques such as SIC. In further works, it would be crucial to study these models behaviors in the long term and to implement different fairness mechanisms to evaluate their performance.

\section{REFERENCES}

[1] ETSI, "EN301 545-2 v1.2.1: Second Generation DVB Interactive Satellite System (DVB-RCS2)."

[2] U. Y. Ng, A. Kyrgiazos, and B. Evans, "Interference coordination for the return link of a multibeam satellite system," in 2014 7th Advanced Satellite Multimedia Systems Conference and the 13th Signal Processing for Space Communications Workshop (ASMS/SPSC), Sep. 2014.

[3] J. Lei and M. A. Vazquez-Castro, "Joint Power and Carrier Allocation for the Multibeam Satellite Downlink with Individual SINR Constraints," in 2010 IEEE International Conference on Communications (ICC).

[4] S. Dimitrov, S. Erl, B. Barth, S. Jaeckel, A. Kyrgiazos, and B. G. Evans, "Radio resource management techniques for high throughput satellite communication systems," in 2015 European Conference on Networks and Communications (EuCNC), Jun. 2015.

[5] G. Cocco, T. D. Cola, M. Angelone, and Z. Katona, "Radio resource management strategies for dvb-s2 systems operated with flexible satellite payloads," in 2016 8th Advanced Satellite Multimedia Systems Conference and the 14th Signal Processing for Space Communications Workshop (ASMS/SPSC), Sept 2016.

[6] V. D'Amico and J. Giese, "ARTIST4g: Innovative scheduling and crosslayer design techniques for interference avoidance," Tech. Rep.

[7] A. Pietrabissa and A. Fiaschetti, "Dynamic uplink frame optimization with adaptive coding and modulation in DVB-RCS2 satellite networks," Int. J. Satell. Commun. Network., vol. 31, no. 3, May 2013.

[8] J.-B. Dupe, Ordonnancement et gestion des ressources pour un système de télécommunications haut débit : Optimisation de la bande passante satellite. Toulouse, INPT, Oct. 2015.

[9] V. Boussemart, M. Berioli, and F. Rossetto, "User scheduling for large multi-beam satellite MIMO systems," in 2011 Conference Record of the Forty Fifth Asilomar Conference on Signals, Systems and Computers (ASILOMAR), Nov. 2011.

[10] D. Lopez-Perez, A. Ladanyi, A. Jüttner, H. Rivano, and J. Zhang, "Optimization Method for the Joint Allocation of Modulation Schemes, Coding Rates, Resource Blocks and Power in Self-Organizing LTE Networks," in 30th IEEE International Conference on Computer Communications (IEEE INFOCOM 2011) mini-conf, Shanghai, China, Apr. 2011.

[11] I. Gurobi Optimization, "Gurobi optimizer reference manual," 2016. 